\title{
Peripartum use of Extracorporeal Membrane Oxygenation (ECMO) in a Patient Suffering from COVID-19 Severe Acute Respiratory Distress Syndrome (ARDS): A Case Report
}

Jay Conhaim ${ }^{1}$, Braxton Forde ${ }^{1}$, Beth Weishaupt ${ }^{1}$, Kara Markham ${ }^{1}$, William Hurford ${ }^{1}$, and C Pickard-Gabriel ${ }^{1}$

${ }^{1}$ University of Cincinnati Medical Center

April 27, 2020

Peripartum use of Extracorporeal Membrane Oxygenation (ECMO) in a Patient Suffering from COVID-19 Severe Acute Respiratory Distress Syndrome (ARDS): A Case Report

Jay Conhaim, $\mathrm{MD}^{1}$, Braxton Forde, $\mathrm{MD}^{2}$, Beth Weishaupt ACNP-BC ${ }^{1}$, Kara Markham, MD², William Hurford, $\mathrm{MD}^{1}, \mathrm{C}$ Jesse Pickard-Gabriel, $\mathrm{MD}^{1}$

1: Department of Anesthesia and Critical Care Medicine, University of Cincinnati Medical Center, Cincinnati, OH USA 45267

2: Division of Maternal-Fetal Medicine, Department of Obstetrics and Gynecology, University of Cincinnati Medical Center, Cincinnati, OH USA 45267

Short title - The Role of VV ECMO in Severe Peripartum COVID-19

Corresponding Author: C Jesse Pickard-Gabriel

Address: Department of Anesthesia and Critical Care, Medical Sciences Building Room 3502, 231 Albert Sabin Way, PO Box 670531, Cincinnati, OH, USA, 45267-0531.

Phone: 513-558-2402

Email: PickardCJ@ucmail.uc.edu

Keywords: ECMO, Pregnant, COVID-19, ARDS

Abstract

We present a 27 year old G2P1001 woman at 29 weeks and 0 days with coronavirus disease 2019 (COVID-19) and subsequent developed of severe ARDS. Following tracheal intubation and hospitalization, the patient and fetus were monitored for seven days. Secondary to worsening oxygenation that was refractory to ventilator and positioning changes, a cesarean was performed at 30 weeks and 0 days with immediate veno-venous (VV) extracorporeal membrane oxygenation (ECMO) cannulation. The patient was transferred to an ECMO referral center and was able to be successfully decannulated and extubated. This discussion focuses on COVID-19 and ARDS in pregnancy with the treatment therein.

Tweetable abstract:

Successful use of VV ECMO to treat severe peripartum COVID-19 PNA in a 27 year old G2P1001 at 29 weeks gestation.

Glossary of Terms 
ARDS - Acute Respiratory Distress Syndrome

AFE - Amniotic Fluid Embolism

COVID-19 - Corona Virus Disease 2019

ECMO - Extracorporeal Membrane Oxygenation

$\mathrm{FdO}_{2}-$ Fraction of Delivered Oxygen (via ECMO)

FiO2 - Fraction of Inspired Oxygen (via ventilator)

PEEP - Positive End Expiratory Pressure

SARS-CoV-2 - Severe Acute Respiratory Syndrome Coronavirus 2

$\mathrm{SpO}_{2}$ - Oxygen Saturation

VV - Veno Venous

Introduction

The utilization of extracorporeal membrane oxygenation (ECMO) in the setting of severe acute respiratory distress syndrome (ARDS) due to coronavirus disease 2019 (COVID-19) infection has been reported in limited numbers. As of this writing the Extracorporeal Life Support Organization has registered 364 confirmed COVID-19 cases on ECMO with $36 \%$ survival to discharge ${ }^{1}$ and there have been no reports of ECMO use in the peripartum setting for severe COVID-19 pneumonia. This case discussion focuses on the peripartum considerations of veno-venous (VV) ECMO for a patient who developed respiratory failure due to Severe Acute Respiratory Syndrome Coronavirus 2 (SARS-CoV-2) infection and subsequent development of COVID-19. Heath Insurance Portability and Accountability Act authorization has been obtained from the patient.

Case

A 27 year-old G2P1001 woman who tested positive for SARS-CoV-2 subsequently developed COVID-19 and was admitted to the hospital three days later. She was 28 weeks and 3 days pregnant by 8 -week ultrasound at time of diagnosis and was initially being monitored at home. On the day of admission, she had developed acute shortness of breath and mild tachycardia, but remained afebrile. Despite $15 \mathrm{l} / \mathrm{min}$ oxygen via a non-rebreather mask, she was unable to maintain oxygen saturations $\left(\mathrm{SpO}_{2}\right)$ above $92 \%$. Her trachea was intubated, and she was transferred to the intensive care unit. She was thrombocytopenic $(73,000 / \mu l)$, lymphopenic $(500 / \mathrm{mL}, 12 \%)$, and mildly anemic (hemoglobin of $11.1 \mathrm{~g} / \mathrm{dl}$ ). C-reactive protein was slightly elevated $(5.3 \mathrm{mg} / \mathrm{l})$ as was interleukin- $6(11.48 \mathrm{pg} / \mathrm{ml})$. A chest radiograph demonstrated bilateral mild atelectasis and mild opacification of the right lung base. The obstetric service diagnosed pre-eclampsia without severe features on the basis of mild hypertension and a protein to creatinine ratio that was elevated to 0.35 . Magnesium therapy was deferred given her respiratory status. Continuous tocometry and fetal heart rate monitoring demonstrated a normal fetal heart rate without regular contractions.

Despite transient improvement and extubation on Day 2, she worsened and required re-intubation on Day 3 for recurrent hypoxemic respiratory failure. There was concern for developing fetal hypoxemia, although three times daily fetal nonstress tests were reassuring. Hydroxychloroquine was administered on Days 3-4, and remdesivir was initiated on Day 5. Her hypoxemia continued to worsen despite fraction of inspired oxygen (FiO2) 1.0, positive end-expiratory pressure (PEEP) of $12 \mathrm{~cm} \mathrm{H2O}$, chemical paralysis, and prone positioning.

Due to significantly worsening respiratory status, a cesarean delivery was performed on Day 7 (30 weeks gestation) prior to completion of a full course of betamethasone therapy for fetal lung maturity. Immediately following an uneventful delivery, the patient received a heparin loading dose. A 25-French right femoral drainage cannula and a 19-French right internal jugular return cannula were placed. Initiation of veno-venous (VV) ECMO improved the $\mathrm{SpO}_{2}$ to $100 \%$. The uterus was closed, however given immediate initiation of 
anticoagulation, the abdomen was temporarily closed with a vacuum-assisted dressing to better monitor hemodynamics and intraabdominal bleeding. The patient received a dose of human convalescent serum and was transferred to an ECMO referral center for further care.

At the referral center, the patient was supported with VV ECMO flows of $4.19 \mathrm{l} / \mathrm{min}$ (cardiac index of 2.08 $\mathrm{l} / \mathrm{min} / \mathrm{m}^{2}$ ), Fraction of Delivered Oxygen $\left(\mathrm{FdO}_{2}\right)$ of $100 \%$ and a sweep flow of $2 \mathrm{l} / \mathrm{min}$, titrating the $\mathrm{FdO}_{2}$ to keep $\mathrm{SpO}_{2}>85 \%$ and $\mathrm{PaCO} 2+/-5 \mathrm{mmHg}$ of $40 \mathrm{mmHg}$. She did not require ongoing neuromuscular blockade. Pressure-controlled ventilation was continued with a plateau pressure of $24 \mathrm{~cm} \mathrm{H}_{2} \mathrm{O}$, PEEP of 10 $\mathrm{cm} \mathrm{H}_{2} \mathrm{O}$, and a respiratory rate of 14 breaths $/ \mathrm{min}$. Tidal volumes were approximately $350 \mathrm{~mL}(5.4 \mathrm{~mL} / \mathrm{kg}$ ideal body weight.) A repeat SARS-CoV-2 test was sent, which confirmed the diagnosis. Vancomycin and cefepime were begun for empiric coverage of likely bacterial superinfection. Low dose norepinephrine and vasopressin infusions were required to maintain mean arterial blood pressures above $65 \mathrm{mmHg}$ while using a furosemide infusion for diuresis. There was no evidence of ongoing bleeding, and her abdominal wall was closed two days after her cesarean delivery.

On Day 8, $\mathrm{FiO}_{2}$ was decreased to 0.3, and PEEP was decreased to $10 \mathrm{~cm} \mathrm{H}_{2} \mathrm{O}$. ECMO flows remained unchanged and $\mathrm{FdO}_{2}$ was decreased to $90 \%$ with a sweep flow of $2 \mathrm{l} / \mathrm{min}$. On Day 9, ECMO flows remained unchanged, $\mathrm{FdO}_{2}$ was decreased to $35 \%$, and the sweep flow was capped. Ventilator settings remained unchanged while tidal volumes increased to $400 \mathrm{~mL}$. Pan-sensitiveStaphalococcus aureus was cultured from a sputum culture collected on Day 1, and antibiotics were changed to cefazolin monotherapy.

On Day 10, with continued improvement, the patient was successfully decannulated at the bedside after capping the ECMO circuit for $>12 \mathrm{hrs}$. Remdesivir therapy, which was discontinued at the time of transfer for administrative reasons, was re-initiated to complete a 10-day course. Vasoactive infusions were discontinued on Day 12, and the patient was extubated on Day 13, and weaned off oxygen therapy on Day 15.

\section{Comment}

At the time of this report there have been almost 2,000,000 confirmed COVID-19 cases worldwide with 126,140 confirmed deaths in 213 countries $^{2}$. The natural history, pathophysiology, epidemiology and associated facets of COVID-19 are still being characterized as the pandemic is ongoing. However, it is understood that COVID-19 is known to prey on patients with existing medical co-morbidities ${ }^{3,4}$. COVID-19 is seemingly less symptomatic in the parturient as compared to the general public ${ }^{5}$, which is in stark contrast to the effects of SARS-CoV-1 and MERS-CoV on pregnant women ${ }^{6}$. The physiologic changes of pregnancy would seemingly engender an increased risk from a SARS-CoV-2 infection. The decrease in functional residual capacity during pregnancy, as well as decreased chest wall and lung compliance in the third trimester ${ }^{7}$, might limit the ability of pregnant women to compensate in the setting of SARS-CoV-2 associated lung disease. Early reports, however, suggest a decreased mortality risk in the parturient ${ }^{5}$. It is hypothesized that a hormone-mediated shift towards T-helper 2 cell-mediated immunity during pregnancy may result in an anti-inflammatory response which could ultimately have protective effects against SARS-CoV- $2^{8}$.

Elevated $\mathrm{SpO}_{2}$ targets to preserve placental oxygenation and avoid placental vasoconstriction necessitate greater levels of oxygen support in pregnant women with $\mathrm{ARDS}^{9}$. The absence of fetal distress and ability to maintain adequate maternal oxygenation initially informed the patient's trajectory of care, however, worsening maternal hypoxemia resulted in fetal delivery as her ability to maintain adequate oxygenation waned despite appropriate positioning maneuvers ${ }^{10,11}$.

The decision to delay delivery of the fetus until the mother wasin extremis directly led to the decision to initiate ECMO at the time of delivery. The mother clearly met criteria for VV ECMO initiation in the hours leading up to delivery, but she improved in short order following delivery and met criteria for decannulation within 24hrs. This raises the obvious question of whether VV ECMO was necessary. Could ECMO have been avoided if the patient had been delivered sooner? Should the medical team have waited to see if delivery improved pulmonary status enough to obviate the need for VV ECMO?

It seems logical that delivery of the fetus generally improves the respiratory status of a mechanically ventilated 
mother, however, this effect may only be seen in severe respiratory disease ${ }^{11}$ and there may be limited benefit if tracheal intubation has occurred due to non-respiratory pathology ${ }^{12,13}$. The highest benefit is seen in instances of mechanical ventilation for obstetric reasons ${ }^{14}$. Additionally, fluid shifts at the time of delivery, often worse in mothers with cardio-pulmonary problems and/or preeclampsia, can lead to poor outcomes. Other uncommon but acute peri-partum complications such as hemorrhage and amniotic fluid embolism (AFE) can be life-threatening in the absence of existing pathology. This patient had no pre-existing cardiac problems, but the additional pulmonary burden of fluid shifts could have proven catastrophic given her predelivery $\mathrm{PaO}_{2}$. The mother's inability to compensate for additional complications such as severe hemorrhage or AFE, justifies planning to cannulate as a safe and reasonable approach. Furthermore, given her high risk of postpartum hemorrhage with necessary initiation of anticoagulation, leaving the abdomen open afterwards, while beneficial for close monitoring and bleeding management, could have likely been avoided if delivery had occurred sooner in the hospital course. Regarding neonatal benefits of delivery, prolonged ventilator support has been associated with increased rates of perinatal asphyxia and while that scenario was unlikely in this case due to the reassuring fetal tracing, expedited delivery is always a consideration ${ }^{15}$.

The timing of delivery and initiation of ECMO in such a critically ill parturient with COVID-19 obviously is not well established. Had the mother been delivered prior to meeting criteria for VV ECMO cannulation, she may have avoided the need for cannulation. However, it is not easy to balance the needs of the mother with the fetus in the setting of a novel and often deadly disease. While this neonate has done well postnatal, consideration for earlier administration of antenatal corticosteroids is important to achieve maximal benefit, as this patient did not receive her first dose of antenatal steroids until the morning of delivery. Regarding the mother, the decision to plan for VV ECMO cannulation following delivery was likely the safest option for the patient by the time that she delivered. While it is possible that the patient would have improved over the two to four hours following delivery, the decision to cannulate immediately was well tolerated and ultimately may have been life-saving for this patient. VV ECMO seems to be a reasonable and feasible option as a life-saving therapy in the setting of severe ARDS and peripartum COVID-19 PNA.

Special Thanks To - Dr. Alex Kobzik, for providing care to this patient prior to transfer and help in gathering data about her course.

Disclosure of Interests:

The authors report no financial disclosures or conflicts of interest related to this project.

Contribution to authorship:

Jay Conhaim: This author prepared the initial manuscript and compiled references.

Beth Weishaupt: This author gathered data and lab results from the both hospitals and helped to build the timeline leading up to cannulation.

Braxton Forde: This author edited the manuscript, contributing to the evaluation and discussion of the peripartum management of the mother and baby.

William Hurford: This author edited the manuscript and provided specific critical-care related insight.

Kara Markham: This author edited the manuscript and comment with specific regards to the peripartum considerations of mother and baby.

C Jesse Pickard-Gabriel (corresponding author): This author edited the manuscript and wrote the comment.

Details of Consent:

We affirm that written consent from the patient was obtained for permission to write and submit this case report.

Funding:

No funding was received by any of the authors for the work provided related to this project. 


\section{References}

1. Extracorporeal Life Support Organization [internet]. https://www.elso.org/Home.aspx. accessed 2020 Apr 16.

2. World Health Organization [internet]. https://www.who.int/emergencies/diseases/novel-coronavirus2019. accessed 2020 Apr 16.

3. Ruan Q, Yang K, Wang W, Jiang L, Song J. Clinical predictors of mortality due to COVID-19 based on an analysis of data of 150 patients from Wuhan, China. Intensive Care Med. 2020; published online Mar 3. DOI:10.1007/s00134-020-06028-z

4. Guan W, Ni Z, Hu Y, et al. Clinical characteristics of coronavirus disease 2019 in China. $N$ Engl J Med. 2020; published online Feb 28. DOI: 10.1056/NEJMoa2002032

5. Schwartz D. An analysis of 38 pregnant women with COVID-19, their newborn infants, and maternal fetal transmission of SARS-CoV-2: maternal coronavirus infections and pregnancy outcomes. Arch Pathol Lab Med. 2020; published online Mar 17. DOI: 10.5858/arpa.2020-0901-SA

6. Wong SF, Chow KM, Leung TN, et al. Pregnancy and perinatal outcomes of women with severe acute respiratory syndrome. Am J Obstet Gynecol. 2004; 191(1):292-7.

7. Gardner MO, Doyle NM. Asthma in pregnancy. Obstet Gynecol Clin North Am. 2004; 31(2):385-413.

8. Dashraath P, Jing Lin Jeslyn W, Mei Xian Karen L, Li Min L, Sarah L, Biswas A, Arjandas Choolani M, Mattar C, Lin SL. Coronavirus disease 2019 (COVID-19) pandemic and pregnancy. Am J Obstet Gynecol.2020; published online Mar 23. DOI: 10.1016/j.ajog.2020.03.021.

9. Dharani K, Narendra DM, Kalpalatha KG. Acute respiratory distress syndrome in pregnancy. In: Jeffrey P, Phelan LDP, Michael R. Foley, George R. Saade, Gary A Dildy, and Michael A. Belfort, eds. Critical Care Obstetrics. Wiley- Blackwell; 2019:403-418.

10. Schwaiberger D, Karcz M, Menk M, Papadakos P, Dantoni S. Respiratory Failure and Mechanical Ventilation in the Pregnant Patient. Crit Care Clin. 2016; 32(1):85-95

11. Lapinsky SE, Rojas-Suarez JA, Crozier TM, Vasquez DN, Barrett N, Austin K, Plotnikow GA, Orellano $\mathrm{K}$, Bourjeily G. Mechanical ventilation in critically-ill pregnant women: a case searies.Int $J$ Obstet Anesth. 2015; 24(4):323-8

12. Tomlinson MW, Caruthers TJ, Whitty JE, Gonik B. Does delivery improve maternal condition in the respiratory-compromised gravida? Obstet Gynecol. 1998; 91(1):108-11.

13. Daily WH, Katz AR, Tonnesen A, Allen SJ. Beneficial effect of delivery in a patient with adult respiratory distress syndrome. Anesthesiology . 1990; 72(2):383-6.

14. Hung CY, Hu HC, Chiu LC, Chang CH, Li LF, Huang CC, Kao CC, Chen PJ, Kao KC. Maternal and neonatal outcomes of respiratory failure during pregnancy. Journal of the Fomosan Medical Association. 2018; 118:413-420.

15. Catanzarite V, Wilms D, Wong D, Landers C, Cousins L, Schrimmer D. Acute Respiratory Distress Syndrome in Pregnancy and the Puerperium: Causes, Courses, and Outcomes. Obstet Gynecol. 2001; 97(5):760-764. 